\title{
Optimization of Cationic SLN for Gene Delivery
}

\author{
S. DoKTOROVOVA ${ }^{1}$, A. M. SILVA ${ }^{2}$, C. M. LOPES ${ }^{1,3}$, \\ P. MARTINS-LOPES ${ }^{1}$, E. B. SOUTO ${ }^{1,3}$ \\ ${ }^{1}$ Institute of Biotechnology and Bioengineering, Centre of Genomics and Biotechnology University of Trás- \\ os-Montes and Alto Douro (IBB/CGB-UTAD), Vila-Real, Portugal \\ ${ }^{2}$ Centre for the Research and Technology of Agro-Environmental and Biological Sciences, University of \\ Trás-os-Montes and Alto Douro (CITAB-UTAD), Vila-Real, Portugal \\ ${ }^{3}$ Department of Pharmaceutical Technology, Faculty of Health Sciences, Fernando Pessoa University, \\ Porto, Portugal \\ E-mails: d0450@utad.eu (S. Doktorovova), eliana@ufp.edu.pt (E. B.Souto)
}

Sci Pharm. 2010; 78: $561 \quad$ doi:10.3797/scipharm.cespt.8.LDD07

Cationic SLN formulations were developed and optimized in terms of cationic lipid/surfactant ratio and production parameters. 5wt\% of Compritol 888 ATO (COM, Gattefosse) or Imwitor 900(IMW,BASF) were used, 0.4-1.0wt\% of cetyltrimethylammonium bromide (CTAB, Sigma) and $0.1-1.0 \mathrm{wt} \%$ of Lutrol F68 (BASF) were tested. Formulations were produced in triplicate. SLN were prepared by modified microemulsion method [1], which was further optimized in terms of velocity and time of high shear homogenization step. Freeze-drying was performed on DuraDry MP at $-45^{\circ} \mathrm{C} / 184 \mathrm{mT}$ during 6 days. Samples were freeze-dryed with $5.0 \%$ or $10.0 \%$ of Glucose (Sigma) or Sucrose (BDH Chemicals) or without cryoprotectants. Reconsitution was performed by rehydratation, vortexing during $3 \mathrm{~min}$ and applying ultrasound during 30s. A Zetasizer NanoSeries (Malvern) was used to determine average hydrodynamic diameter (z-ave), polydispersity (PdI) and zeta potential (ZP)

Optimized formulation consisted of $5.0 \%$ solid lipid, $0.5 \%$ CTAB and $0.25 \%$ Lutrol F68, yielding particles with $Z$-ave $=159 \mathrm{~nm}, \mathrm{Pdl}=0.34$ and $\mathrm{ZP}=54.8 \mathrm{mV}$ (IMW) and $Z$-ave=180nm, Pdl=0.272 and $Z P=56.2 \mathrm{mV}(\mathrm{COM})$. These parameters remained unchanged during 8 days of storage at $8^{\circ} \mathrm{C}$. Samples dried without cryoprotectants yielded light powdery product which could be redispersed easily; freeze-dried samples with saccharides tested yielded solid products.

Cationic SLN formulations intended for gene delivery were designed and optimized. Particles with z-ave below 200nm and low Pdl were produced. These SLN showed good stability of 15 days .Suitability of cationic SLN for freezedrying without cryoprotectants was confirmed [2].

The authors whish to thank Dr. Fernando Nunes for cooperation in freezedrying experiments. This work was supported by grant SFRH/BD/60552/2009 from FCT and by Nadacia Slovenskeho Plynarenskeho Priemyslu.

[1] Shah KA, Joshi MD, Patravale VB. Biocompatible Microemulsions for Fabrication of Glyceryl Monostearate Solid Lipid Nanoparticles (SLN) of Tretinoin. J Biomed Nanotechnol. 2009; 5: 396-400. doi:10.1166/jbn.2009.1048

[2] Vighi E, Ruozi B, Montanari M, Battini R, Leo E. Re-dispersible cationic solid lipid nanoparticles (SLNs) freeze-dried without cryoprotectors: Characterization and ability to bind the pEGFP-plasmid. Eur J Pharm Biopharm. 2007; 67: 320-328. doi:10.1016/j.ejpb.2007.02.006 